\title{
Associations between maternal mid-pregnancy apolipoprotein A-1, apolipoprotein B, apolipoprotein B/apolipoprotein A-1 ratio and preterm birth: An observational study
}

\author{
Qing Liu ${ }^{1}$, Li Wu ${ }^{2}$, Lulin Wang ${ }^{1}$, Lulu Song ${ }^{1}$, Yunyun Liu ${ }^{1}$, Mingyang $\mathrm{Wu}^{1}$, Jianing $\mathrm{Bi}^{1}$, \\ Kai Chen ${ }^{1}$, Yuntao $\mathrm{Wu}^{2}$, Jianhong $\mathrm{Xia}^{2}$, and Youjie Wang ${ }^{1}$ \\ ${ }^{1}$ Huazhong University of Science and Technology Tongji Medical College \\ ${ }^{2}$ Guangdong Women and Children Hospital
}

February 26, 2021

\begin{abstract}
Objective: To investigate the association between maternal apolipoprotein A-1 (ApoA-1), apolipoprotein B (ApoB), ApoB/ApoA1ratio and preterm birth (PTB). Design: Observational survey. Setting: Guangdong Women and Children Hospital, China Samples: 5986 pregnant women. Method: Data were extracted from the information system of Guangdong Women and Children Hospital. Maternal serum ApoB, ApoA-1 and ApoB/ApoA-1 ratio were categorized into tertiles. Logistic regression models were performed to evaluate the odds ratios and $95 \%$ confidence intervals for PTB. Main outcome measures: PTB (gestational age $<37$ weeks) Results: Of the study participants, the rate of preterm birth was 5.7\% ( $\mathrm{n}=344)$. Compare with individuals in the lowest tertile, the adjusted ORs of PTB was 1.41 (95\% CI: 1.07-1.87) for individuals in the highest tertile of ApoB and 1.35 (95\% CI: 1.03-1.78) for those in the highest tertile of ApoB/ApoA-1. The association of ApoB/ApoA-1 and PTB was more relevant among women with pre-pregnancy BMI [?] $24 \mathrm{~kg} / \mathrm{m} 2$, age at delivery [?] 35 years or those who were multiparous. Conclusions: Elevated maternal ApoB level and ApoB/ApoA-1 ratio during mid-pregnancy were related to increased risk of PTB. Monitoring serum apolipoprotein levels may help to prevent preterm birth. Funding: The study was funded by grants 2019FYH003 from Chinese Center for Disease Control and Prevention. Keywords: Apolipoprotein A-1, Apolipoprotein B, Apolipoprotein B/Apolipoprotein A-1, Preterm birth Tweetable abstract:Maternal mid-pregnancy ApoB level and ApoB/ApoA-1 ratio were associated with PTB.
\end{abstract}

\section{INTRODUCTION}

Preterm birth (PTB), defined as birth before 37 complete gestational weeks, accounts for about $18 \%$ of all deaths in under-five children and $35 \%$ of death among newborns ${ }^{1}$. Evidence have suggested that PTB was related to a serious of adverse health outcomes during childhood and adulthood, such as neurodevelopmental impairment, respiratory diseases, diabetes mellitus, hypertension and cardiovascular disease $\mathrm{s}^{2-6}$. Precise etiology of PTB is still largely unknown. Emerging evidence has suggested that maternal dyslipidemia during pregnancy might be a risk factor for $\mathrm{PTB}^{7-13}$. A recent meta-analysis of these studies reported that a more atherogenic maternal lipid profile including elevated total cholesterol, triglycerides and low high-density lipoprotein (HDL) was positively related to the risk of $\mathrm{PTB}^{14}$.

Apolipoprotein (Apo) is a key component of lipid metabolism and transportation. ApoA-1 is the main structural protein component of HDL. ApoB represents the total amount of potentially atherogenic circulating lipoproteins, including low-density lipoprotein (LDL), very low-density lipoprotein (VLDL) and intermediate-density lipoproteins (IDL). The ApoB/ApoA-1 ratio reflects cholesterol balance between atherogenic and anti-atherogenic lipoprotein particles and were thought to be a reliable parameter to reflect lipid disturbance $^{15}$. Abundant evidence have shown that apolipoprotein levels were associated with metabolic 
syndrome $^{16}$, insulin resistance ${ }^{17}$ and cardiovascular disease ${ }^{15}$ among non-pregnant population. Epidemiological studies suggested that serum ApoB level and ApoB/ApoA-1 ratio during pregnancy were positively related to the risk of preeclampsia ${ }^{18-20}$, uteroplacental acute atherosis ${ }^{21}$ and intrauterine growth restriction ${ }^{22}$. However, evidence regarding the association between maternal apolipoprotein levels and PTB is limited. To our knowledge, only three previous studies have investigated the relationship between maternal ApoA-1 and PTB with conflicting results ${ }^{23-25}$. Furthermore, the effects of maternal ApoB and ApoB/ApoA-1 ratio on PTB have not been studied yet.

Thus, we aim to explore the relationship between serum ApoA-1, ApoB, ApoB/ApoA-1 ratio during pregnancy and PTB among Chinese pregnant women.

\section{MATERIALS AND METHODS}

\section{Data source and study population}

Births were identified from the records collected by the information system of Guangdong Women and Children Hospital from September 2014 to March 2018 ( $\mathrm{N}=37,193)$. We extracted maternal information including age at delivery, delivery mode, pre-pregnancy weight and height, parity, the date of last menstrual period, delivery date as well as smoking status during pregnancy. We also extracted the information of the newborns including birth weight and infant sex. The test results of maternal mid-pregnancy ApoA-1, ApoB and oral glucose tolerance test (OGTT) were obtained from laboratory information system of the study hospital. Maternal mid-pregnancy ApoA-1 and ApoB values were determined based on fasting blood samples tested during their routine pregnancy tests at a median of 18 weeks of gestation (IQR : $16-22$ weeks) and were measured by immunoturbidimetric assays.

The women satisfying the follow criteria were included in the analysis: 1) who had the data of mid-pregnancy ApoA-1 and ApoB, OGTT, the date of last menstrual period, pre-pregnancy weight and height; 2) gave a single live birth. We excluded post-term births (gestational age $>42$ weeks), babies with birth defects or the recorded birthweight out of the range 500-5000 g. We further excluded the outlier value $(<25$ th $-1.5 \mathrm{IQR}$ or $>75$ th +1.5 IQR) of ApoA-1 and ApoB. Finally, a total of 5,986 mother-newborn pairs were included in the present study (Fig. 1). We compared characteristics between included women $(n=5,986)$ and those who were excluded due to mid-pregnancy apolipoprotein measurements were not available $(n=7,917)$. Mothers in the included group were slightly younger $(32.2 \mathrm{vs} 32.4 ; \mathrm{p}=0.021)$ than women who were excluded but there was no significant difference in pre-pregnancy BMI or the incidence of preterm birth between this two groups (see supplementary Table $\mathbf{S} \mathbf{1}$ ).

As all data used in the present study was de-identified, no written informed consents were obtained from the participants. The study protocol has been approved by the ethics committee of Guangdong Women and Children Hospital.

\section{Assessment of covariates and outcome definition}

Pre-pregnancy body mass index (BMI) was calculated by dividing self-reported pre-pregnancy weight (kg) by height squared $\left(\mathrm{m}^{2}\right)$. According to the International Association of Diabetes and Pregnancy Study Groups (IADPSG) criteria $^{26}$, gestational diabetes mellitus (GDM) was diagnosed when OGTT fasting [?] $5.1 \mathrm{mmol} / \mathrm{L}$ or OGTT 1 hour [?] $10.0 \mathrm{mmol} / \mathrm{L}$ or OGTT 2 hour [?] $8.5 \mathrm{mmol} / \mathrm{L}$. The date of last menstrual period was used to estimate gestational age (in weeks). PTB was defined as gestational week less than 37 weeks.

\section{Statistical analysis}

Baseline characteristics of study population were described for preterm birth and term birth separately. Data were expressed as mean \pm standard deviation (SD) for normally distributed variables or median (interquartile range, IQR) for skewed variable or frequency (percentage) for categorical variable. To test for difference between preterm birth and term birth groups, continuous variables were compared by $t$-test or Wilcoxon test and categorical variables were compared by $C h i$-square test. ApoB, ApoA-1 and ApoB/ApoA-1 were 
categorized into tertiles (ApoA-1: < 1.98, 1.98-2.18 or [?] $2.18 \mathrm{~g} / \mathrm{L} ;$ ApoB: $<0.88,0.88-1.06$ or [?] $1.06 \mathrm{~g} / \mathrm{L}$; ApoB/ApoA-1: $<0.42,0.42-0.52$ or [?] 0.52$)$ and the lowest tertile was used as the reference in the analysis. Logistic regression was used to estimate the odds ratio (ORs) and $95 \%$ confidence intervals (CIs) for the associations between ApoB, ApoA-1, ApoB/ApoA-1 ratio and the risk of PTB. ApoB, ApoA-1and their ratio were included in two ways: categorical or continuous variables. All multivariate models were adjusted for potential confounders including maternal age at delivery (continuous), pre-pregnancy BMI (continuous), parity (primiparous or multiparous), delivery mode (vaginal delivery or cesarean delivery), GDM (yes or no) and infant sex (male or female). Since smoking is rare among Chinese pregnant population and in our study only four women admitted smoking during pregnancy, maternal smoking status were not adjusted. Restricted cubic spline analysis with four knots (5th, 35th, 65th and 95th percentiles) were conducted to further explore non-linear dose-response relationship between mid-pregnancy apolipoprotein profile and risk of preterm birth.

Subgroup analyses were conducted according to maternal pre-pregnancy BMI ([?] $24 \mathrm{~kg} / \mathrm{m}^{2},>24 \mathrm{~kg} / \mathrm{m}^{2}$ ), maternal age at delivery ( $<35$ years, [?] 35 years) and parity (primiparous, multiparous). The cut-off of pre-pregnancy BMI was decided according to the recommendation of the Working Group on Obesity in China ${ }^{27}$.

The presence of pregnancy complications may affect the association between apolipoprotein and preterm birth. Therefore, we performed a sensitivity analysis among a subgroup of 5,080 mother-newborn pairs after excluding mothers with GDM.

All statistical analyses were performed by $\mathrm{R}$ software (version 3.5.3) and SAS (version 9.4; SAS Institute Inc.). $P<0.05$ was considered statistically significant.

\section{RESULTS}

Baseline characteristics of the study population were showed inTable 1. A total of 5,986 mother-newborn pairs were included in the analyses and $5.7 \%(\mathrm{n}=344)$ of them were preterm birth. The mean maternal age at delivery was $32.2+-4.7$ years and the mean pre-pregnancy BMI was $20.7+-2.9 \mathrm{~kg} / \mathrm{m}^{2}$. The median values $(\mathrm{IQR})$ of $\mathrm{ApoB}, \mathrm{ApoA}-1$ and ApoB/ApoA-1 ratio were $2.07(0.30) \mathrm{g} / \mathrm{L}, 0.96(0.28) \mathrm{g} / \mathrm{L}$ and 0.47 (0.14), respectively. The results showed that preterm birth occurred more frequently in those mothers with more adverse risk profiles, including being older at delivery, multiparous, had higher pre-pregnancy BMI, with higher ApoB and ApoB/ApoA-1 levels during second trimester.

The relationships between maternal apolipoprotein levels and PTB were presented in Table 2. In the crude model, participants in the highest tertile of $\mathrm{ApoB}(\mathrm{OR}, 1.54 ; 95 \% \mathrm{CI}, 1.17-2.02 ; P$ for trend $=0.002)$ and ApoB/ApoA-1 (OR, 1.42; 95\% CI, 1.09-1.85; Pfor trend=0.007) had a significantly higher risk of PTB, compared to those in the lowest tertile. Adjustment for potential confounders did not materially alter the positive associations between ApoB, ApoB/ApoA-1 and PTB. Compared with the lowest tertiles, individuals in the highest tertile of $\mathrm{ApoB}$ had a $41 \%$ higher risk of PTB $(\mathrm{OR}, 1.41 ; 95 \%$ CI, 1.07-1.87; $P$ for trend= 0.017) and those in the highest tertile of ApoB/ApoA-1 had a 35\% higher risk of PTB (OR, 1.35; 95\% CI, 1.03-1.78; $P$ for trend= 0.024). There are no differences in the risk of PTB across the ApoA-1 tertiles. When continuous variables were used, each $1-\mathrm{g} / \mathrm{L}$ increase in $\mathrm{ApoB}$ and each 1 unit increase in $\mathrm{ApoB} / \mathrm{ApoA}-1$ were positively related to the risk of PTB (OR, 2.33; 95\% CI 1.40-3.86 for ApoB and OR, 4.10; 95\% CI, 1.47-11.36 for ApoB/ApoA-1). We further conducted restricted cubic spline to estimate the dose-response relationships between apolipoprotein levels and PTB (Fig. 2 ). The results indicated that ApoB ( $P$ for non-linearity $=$ $0.943)$ and ApoB/ApoA-1 ( $P$ for non-linearity $=0.284)$ were both linearly associated with the risk of PTB, whereas ApoA-1 was not associated with PTB $(P$ for all $=0.092)$.

The results of subgroup analyses were shown in Table $\mathbf{3}$. Even though there was no interaction between all of the stratification variables and preterm birth (all $P$ for interaction $>0.05$ ), the positive relationship between maternal ApoB/ApoA-1 and preterm birth was stronger in women with pre-pregnancy BMI [?] 24 $\mathrm{kg} / \mathrm{m}^{2}$, age at delivery [?] 35 years or those who were multiparous. In the sensitivity analyses, the positive relationships between ApoB, ApoB/ApoA-1 ratio during pregnancy and preterm birth were not materially 
changed after excluding pregnant women with GDM (Table 4 ).

\section{DISCUSSION}

\section{Main findings}

To the best of our knowledge, this is the first study to estimate the association between ApoB, ApoB/ApoA-1 ratio during mid-pregnancy and the risk of PTB. We found that maternal mid-pregnancy ApoB level and ApoB/ApoA-1 ratio were positively related to the risk of PTB. In addition, these associations showed a linear dose-response relationship. Subgroup analysis suggested that the effect of ApoB/ApoA-1 ratio might be more relevant among women with pre-pregnancy BMI [?] $24 \mathrm{~kg} / \mathrm{m}^{2}$, age at delivery [?] 35 years or those who were multiparous.

\section{Strengths and limitations}

One of the strengths of this study was its relatively large sample size which made the results more reliable. In addition, compare to other lipid parameters used in former studies such as HDL and LDL, ApoB/ApoA-1 is thought to be a more reliable and comprehensive parameter to reflect lipid disturbance ${ }^{28}$. However, several limitations should also be pointed out. First, pre-pregnancy weight was self-reported which may cause recall bias, although the discrepancy between self-reported weight and measured weight was relatively small ${ }^{29}$. Second, although we adjusted for several potential confounders, we cannot rule out the possibility of residual confounding by unmeasured factors, such as gestational dietary nutrition, gestational weight gain, maternal life style and socioeconomic parameters. Third, the participant of our study were all Chinese pregnant women, which may reduce the generalization of our results to other ethnic groups.

\section{Interpretation}

Elevated ApoB level or ApoB/ApoA-1 ratio represent a more atherogenic lipid profile and was known to be related to several chronic diseases including metabolic syndrome ${ }^{16}$, insulin resistance ${ }^{17}$ and cardiovascular disease ${ }^{15}$ among non-pregnant population. In addition, it has been reported that ApoB/ApoA-1 ratio can predict cardiovascular disease better than any other cholesterol indices ${ }^{28}$. Previous studies have also investigated the relationship between maternal apolipoprotein profile and pregnancy complications. Several studies have demonstrated that $\mathrm{ApoB}$ and $\mathrm{ApoB} / \mathrm{ApoA}-1$ ratio during pregnancy was positively related to the risk of preeclampsia ${ }^{18-20}$. Recently, an animal research found that intravenous infusion of apoA1 could lower pregnancy-induced insulin resistance in rats ${ }^{30}$. But Ravi et al. reported that serum apoA1 level during mid-pregnancy was not associated with insulin resistance or the risk of GDM in pregnant women ${ }^{31}$. However, studies regarding the association between apolipoprotein profile and adverse birth outcome is still scarce so far. Till now, only three studies have explored the association between maternal ApoA-1 and PTB. A nested case-control study conducted in America revealed that ApoA-1 concentration during early pregnancy was positively correlated with the risk of spontaneous preterm birth ${ }^{24}$. However, another case-control study conducted in Australia found that maternal early pregnancy ApoA-1 level was negatively related to the risk of spontaneous preterm birth. ${ }^{23}$. In addition, Abiaka et al. ${ }^{25}$ reported that serum ApoA-1 level during labor was significantly lower in preterm delivery mothers. In the present study, we found no association between maternal ApoA-1 and PTB. The discrepancies in the findings among former studies and ours might be explained partly by the difference in trimester for ApoA-1 testing and difference in racial background for the study participants.

The results of subgroup analyses showed that the positive association between maternal ApoB/ApoA-1 ratio during mid-pregnancy and risk of PTB was more pronounced among participants with pre-pregnancy BMI [?] $24 \mathrm{~kg} / \mathrm{m}^{2}$, age at delivery [?] 35 years or those who were multiparous, although there was no interaction between all of the stratification variables and preterm birth. Prior studies have shown that women with higher pre-pregnancy BMI were more likely to develop pre-eclampsia and diabetes during pregnancy, which greatly increased the risk of $\mathrm{PTB}^{32}$. Primiparity is also known to be a risk factor for $\mathrm{PTB}^{33}$. Therefore, a logical explanation might be that the existence of strong risk factors for PTB such as obesity might mask the effects of ApoB/ApoA-1 on the occurrence of PTB. Maternal ageing is related to increased levels of inflammation 
and oxidative stress ${ }^{34}$ and have an adverse impact on the placenta ${ }^{35}$. Therefore, pregnant women with an advanced age are more vulnerable to the elevation of atherogenic lipoproteins during pregnancy might explain the different results between these two age groups.

There are several mechanisms might explain the increased risk of PTB among women with higher serum ApoB level or ApoB/ApoA-1 ratio. Previous studies have revealed a positive association between ApoB level and oxidative stress ${ }^{36}$. Elevated level of oxidative stress during pregnancy is related to higher risk of $\mathrm{PTB}^{37}$. Furthermore, both experimental ${ }^{38,39}$ and epidemiological studies ${ }^{36,40}$ have reported that elevated ApoB level or ApoB/ApoA-1 ratio was associated with endothelial damage. Chen et al. ${ }^{41}$ reported that impaired endothelial function was present in women with preterm delivery. In addition, previous study have found that competitive inhibition of fibrinolysis by $\mathrm{ApoB}$ were associated with hemorheology modification in intrauterine growth restriction cases and suggested that ApoB/ApoA-1 might be a good marker of early detection of intrauterine growth restriction ${ }^{22}$.

\section{Conclusion}

In conclusion, higher levels of serum ApoB and ApoB/ApoA-1 ratio during mid-pregnancy were associated with an increased risk of PTB among Chinese pregnant women. Monitoring and managing apolipoprotein levels during pregnancy may help to identify the high-risk population and reduce the risk of preterm birth. Further studies are needed to confirm the positive relationships between ApoB, ApoB/ApoA-1 during pregnancy and PTB and to explore the underlying mechanisms.

\section{Acknowledgment}

We sincerely thank all the participants in this study and clinical administrative staff in Guangdong Woman and Children Hospital, China.

\section{Details of ethics statement}

The study protocol has been approved by the ethics committee of Guangdong Women and Children Hospital (permit number 202001225) in December $18^{\text {th }} 2020$.

\section{Disclosure of interests}

The authors report grants from Chinese Center for Disease Control and Prevention. There is no other conflict of interest to declare. Completed disclosure of interest forms are available to view online as supporting information.

\section{Contribution to authorship}

Qing Liu, Li Wu, Youjie Wang, Yuntao Wu, Jianhong Xia contributed to the conception and design of the research. Lulin Wang, Lulu song, Mingyang Wu, Yunyun Liu, Jianing Bi, Kai Chen assisted in data acquisition. Qing liu and $\mathrm{Li} \mathrm{Wu}$ performed the statistical analysis and draft the initial manuscript. Youjie Wang, Yuntao Wu and Jianhong Xia provided content and feedback to the manuscript, reviewed and edited.

\section{Funding}

The study was funded by grants 2019FYH003 from the Chinese Center for Disease Control and Prevention

\section{Reference}

1. UN Inter-Agency Group for Child Mortality Estimation.: Levels and trends in child mortality: Report 2017. New York: United Nations Children's Fund. In.; 2017.

2. Luu TM, Rehman Mian MO, Nuyt AM. Long-Term Impact of Preterm Birth: Neurodevelopmental and Physical Health Outcomes. Clin Perinatol. 2017;44(2):305-314.

3. Crump C, Winkleby MA, Sundquist J, Sundquist K. Risk of asthma in young adults who were born preterm: a Swedish national cohort study. Pediatrics. 2011;127(4):e913-920. 
4. Crump C, Winkleby MA, Sundquist K, Sundquist J. Risk of Hypertension Among Young Adults Who Were Born Preterm: A Swedish National Study of 636,000 Births. Am J Epidemiol. 2011;173(7):797-803.

5. Kerr-Wilson CO, Mackay DF, Smith GCS, Pell JP. Meta-analysis of the association between preterm delivery and intelligence. Journal of Public Health. 2012;34(2):209-216.

6. Tinnion R, Gillone J, Cheetham T, Embleton N. Preterm birth and subsequent insulin sensitivity: a systematic review. Arch Dis Child. 2014;99(4):362-368.

7. Edison RJ, Berg K, Remaley A, Kelley R, Rotimi C, Stevenson RE, et al. Adverse birth outcome among mothers with low serum cholesterol. Pediatrics. 2007;120(4):723-733.

8. Kramer MS, Kahn SR, Rozen R, Evans R, Platt RW, Chen MF, et al. Vasculopathic and thrombophilic risk factors for spontaneous preterm birth. Int J Epidemiol. 2009;38(3):715-723.

9. Mudd LM, Holzman CB, Catov JM, Senagore PK, Evans RW. Maternal lipids at mid-pregnancy and the risk of preterm delivery. Acta Obstet Gyn Scan. 2012;91(6):726-735.

10. Niromanesh S, Shirazi M, Dastgerdy E, Sharbaf FR, Shirazi M, Khazaeipour Z. Association of hypertriglyceridaemia with pre-eclampsia, preterm birth, gestational diabetes and uterine artery pulsatility index. Natl Med J India. 2012;25(5):265-267.

11. Vrijkotte TG, Krukziener N, Hutten BA, Vollebregt KC, van Eijsden M, Twickler MB. Maternal lipid profile during early pregnancy and pregnancy complications and outcomes: the ABCD study. J Clin Endocrinol Metab. 2012;97(11):3917-3925.

12. Emet T, Ustuner I, Guven SG, Balik G, Ural UM, Tekin YB, et al. Plasma lipids and lipoproteins during pregnancy and related pregnancy outcomes. Archives of Gynecology and Obstetrics. 2013;288(1):49-55.

13. Maymunah AO, Kehinde O, Abidoye G, Oluwatosin A. Hypercholesterolaemia in pregnancy as a predictor of adverse pregnancy outcome. African Health Sciences. 2014;14(4).

14. Jiang SY, Jiang JX, Xu HW, Wang SJ, Liu ZY, Li MC, et al. Maternal dyslipidemia during pregnancy may increase the risk of preterm birth: A meta-analysis. Taiwan J Obstet Gyne. 2017;56(1):9-15.

15. Kappelle PJWH, Gansevoort RT, Hillege JL, Wolffenbuttel BHR, Dullaart RPF, Grp PS. Apolipoprotein B/A-I and total cholesterol/high-density lipoprotein cholesterol ratios both predict cardiovascular events in the general population independently of nonlipid risk factors, albuminuria and C-reactive protein. J Intern Med. 2011;269(2):232-242.

16. Sierra-Johnson J, Somers VK, Kuniyoshi FHS, Garza CA, Isley WL, Gami AS, et al. Comparison of apolipoprotein-B/apolipoprotein-AI in subjects with versus without the metabolic syndrome. Am J Cardiol. 2006;98(10):1369-1373.

17. Sung KC, Hwang ST. Association between insulin resistance and apolipoprotein B in normoglycemic Koreans. Atherosclerosis. 2005;180(1):161-169.

18. Basu A, Alaupovic P, Wu M, Jenkins AJ, Yu Y, Nankervis AJ, et al. Plasma lipoproteins and preeclampsia in women with type 1 diabetes: a prospective study. J Clin Endocrinol Metab. 2012;97(5):1752-1762.

19. Khaire AA TS, Wagh GN, Joshi SR. Placental lipid metabolism in preeclampsia. J Hypertens. 2020.

20. Timur H, Daglar HK, Kara O, Kirbas A, Inal HA, Turkmen GG, et al. A study of serum Apo A-1 and Apo B-100 levels in women with preeclampsia. Pregnancy Hypertens. 2016;6(2):121-125.

21. Moe K, Alnaes-Katjavivi P, Storvold GL, Sugulle M, Johnsen GM, Redman CWG, et al. Classical Cardiovascular Risk Markers in Pregnancy and Associations to Uteroplacental Acute Atherosis. Hypertension. 2018;72(3):695-702. 
22. Munoz A, Uberos J, Molina A, Valenzuela A, Cano D, Ruiz C, et al. Relationship of blood rheology to lipoprotein profile during normal pregnancies and those with intrauterine growth retardation. J Clin Pathol. $1995 ; 48(6): 571-574$.

23. D'Silva AM, Hyett JA, Coorssen JR. First Trimester Protein Biomarkers for Risk of Spontaneous Preterm Birth: Identifying a Critical Need for More Rigorous Approaches to Biomarker Identification and Validation. Fetal Diagn Ther. 2020;47(6):497-506.

24. Chen X, Scholl TO, Stein TP, Steer RA, Williams KP. Maternal Circulating Lipid Profile during Early Pregnancy: Racial/Ethnic Differences and Association with Spontaneous Preterm Delivery. Nutrients. $2017 ; 9(1)$.

25. Abiaka C, Machado L, Mathew M, Rao K. Erythrocyte indices, microminerals and ratios, antioxidants and lipids in Centrum Materna diet-supplemented Omani mothers. Biol Trace Elem Res. 2008;124(1):83-91.

26. Metzger BE, Gabbe SG, Persson B, Buchanan TA, Catalano PM, Damm P, et al. International Association of Diabetes and Pregnancy Study Groups Recommendations on the Diagnosis and Classification of Hyperglycemia in Pregnancy. Diabetes Care. 2010;33(3):676-682.

27. Zhou BF, Cooperative Meta-Analysis Group of the Working Group on Obesity in C. Predictive values of body mass index and waist circumference for risk factors of certain related diseases in Chinese adults-study on optimal cut-off points of body mass index and waist circumference in Chinese adults. Biomed Environ Sci. 2002;15(1):83-96.

28. Sierra-Johnson J, Fisher RM, Romero-Corral A, Somers VK, Lopez-Jimenez F, Ohrvik J, et al. Concentration of apolipoprotein B is comparable with the apolipoprotein B/apolipoprotein A-I ratio and better than routine clinical lipid measurements in predicting coronary heart disease mortality: findings from a multi-ethnic US population. Eur Heart J. 2009;30(6):710-717.

29. Headen I, Cohen AK, Mujahid M, Abrams B. The accuracy of self-reported pregnancy-related weight: a systematic review. Obes Rev. 2017;18(3):350-369.

30. Wu BJ, Sun Y, Ong KL, Li Y, Tang S, Barter PJ, et al. Apolipoprotein A-I Protects Against PregnancyInduced Insulin Resistance in Rats. Arterioscler Thromb Vasc Biol. 2019;39(6):1160-1171.

31. Retnakaran R, Ye C, Connelly PW, Hanley AJ, Sermer M, Zinman B. Serum apoA1 (Apolipoprotein A-1), Insulin Resistance, and the Risk of Gestational Diabetes Mellitus in Human Pregnancy-Brief Report. Arterioscler Thromb Vasc Biol. 2019;39(10):2192-2197.

32. Goldenberg RL, Culhane JF, Iams JD, Romero R. Epidemiology and causes of preterm birth. Lancet. 2008;371(9606):75-84.

33. Koullali B, van Zijl MD, Kazemier BM, Oudijk MA, Mol BWJ, Pajkrt E, et al. The association between parity and spontaneous preterm birth: a population based study. Bmc Pregnancy Childb. 2020;20(1).

34. Syslova K, Bohmova A, Mikoska M, Kuzma M, Pelclova D, Kacer P. Multimarker screening of oxidative stress in aging. Oxid Med Cell Longev. 2014;2014:562860.

35. Lean SC, Heazell AEP, Dilworth MR, Mills TA, Jones RL. Placental Dysfunction Underlies Increased Risk of Fetal Growth Restriction and Stillbirth in Advanced Maternal Age Women. Sci Rep. 2017;7(1):9677.

36. Emoto T, Sawada T, Morimoto N, Tenjin T, Wakimoto T, Ikeda F, et al. The apolipoprotein B/A1 ratio is associated with reactive oxygen metabolites and endothelial dysfunction in statin-treated patients with coronary artery disease. J Atheroscler Thromb. 2013;20(7):623-629.

37. Moore TA, Ahmad IM, Zimmerman MC. Oxidative Stress and Preterm Birth: An Integrative Review. Biol Res Nurs. 2018;20(5):497-512. 
38. Zinellu A, Sotgia S, Scanu B, Pintus G, Posadino AM, Cossu A, et al. S-homocysteinylated LDL apolipoprotein B adversely affects human endothelial cells in vitro. Atherosclerosis. 2009;206(1):40-46.

39. Zhang Y, Zhang W, Edvinsson L, Xu CB. Apolipoprotein B of low-density lipoprotein impairs nitric oxide-mediated endothelium-dependent relaxation in rat mesenteric arteries. Eur J Pharmacol. 2014;725:1017.

40. Karasek D, Vaverkova H, Cibickova L, Gajdova J, Kubickova V. Apolipoprotein B vs non-high-density lipoprotein cholesterol: Association with endothelial hemostatic markers and carotid intima-media thickness. J Clin Lipidol. 2017;11(2):442-449.

41. Chen X, Scholl TO. Maternal biomarkers of endothelial dysfunction and preterm delivery. PLoS One. 2014;9(1):e8

\section{Legends for Figures and Tables}

Table 1. Characteristics of the 5,986 mother-newborn pairs in the present study

Table 2. Odds ratios and $95 \%$ confidence intervals for preterm birth according to maternal ApoA-1, ApoB and ApoB/ApoA-1.

Table 3. Subgroup analyses of the associations between ApoB, ApoB/ApoA-1 and preterm birth, stratified by pre-pregnancy BMI, maternal age and parity.

Table 4. Sensitivity analyses: odds ratios and $95 \%$ confidence intervals for preterm birth according to maternal serum ApoA-1, ApoB and ApoB/ApoA-1.

Figure 1. Flowchart of the study population.

Figure 2. Relationship between ApoA-1, ApoB, ApoB/ApoA-1 and preterm birth, fitted by restricted cubic splines. Odds ratio were estimated using logistic regression model adjusting for maternal pre-pregnancy BMI, age at delivery, parity, delivery mode, gestational diabetes mellitus and infant sex. The solid line represents odds ratios and the dash area are $95 \%$ confidence intervals. Four knots were located at 5th, 35th, 65th 95th percentiles of ApoA-1, ApoB, ApoB/ApoA-1.

Table 1. Characteristics of the 5,986 mother-newborn pairs in the present study

\begin{tabular}{|c|c|c|c|c|}
\hline Characteristics & Total $(\mathrm{N}=5,986)$ & $\begin{array}{l}\text { Preterm birth }(\mathrm{N} \\
=344)\end{array}$ & $\begin{array}{l}\text { Term birth }(\mathrm{N}= \\
5,642)\end{array}$ & $P$ Value \\
\hline \multicolumn{5}{|l|}{ Maternal } \\
\hline \multicolumn{5}{|l|}{ Characteristics } \\
\hline $\begin{array}{l}\text { Maternal age at } \\
\text { delivery, years } \\
\text { Parity }\end{array}$ & $32.2 \pm 4.7$ & $33.4 \pm 5.0$ & $32.2 \pm 4.7$ & $<0.001$ \\
\hline Primiparous & $2124(35.5)$ & $107(31.1)$ & $2017(35.7)$ & $<0.001$ \\
\hline Multiparous & $3862(64.5)$ & $237(68.9)$ & $3625(64.3)$ & \\
\hline $\begin{array}{l}\text { Pre-pregnancy } \\
\text { BMI, } \mathrm{kg} / \mathrm{m}^{2}\end{array}$ & $20.7 \pm 2.9$ & $21.1 \pm 3.0$ & $20.7 \pm 2.9$ & 0.005 \\
\hline $\begin{array}{l}\text { Smoking during } \\
\text { pregnancy }\end{array}$ & & & & $\mathrm{NA}$ \\
\hline Yes & $4(0.1)$ & $0(0.0)$ & $4(0.1)$ & \\
\hline No & $5982(99.9)$ & $344(100.0)$ & $5638(99.9)$ & \\
\hline GDM & & & & $<0.001$ \\
\hline Yes & $906(15.4)$ & $74(21.5)$ & $832(14.7)$ & \\
\hline No & $5080(84.6)$ & $270(78.5)$ & $4810(85.3)$ & \\
\hline $\begin{array}{l}\text { Delivery mode } \\
\text { cesarean delivery }\end{array}$ & $2107(35.2)$ & $164(47.7)$ & $1943(34.4)$ & $<0.001$ \\
\hline
\end{tabular}




\begin{tabular}{|c|c|c|c|c|}
\hline vaginal delivery & $3879(64.8)$ & $180(52.3)$ & $3699(65.6)$ & \\
\hline Neonatal & & & & \\
\hline Characteristics & & & & \\
\hline $\begin{array}{l}\text { Infant sex } \\
\text { male }\end{array}$ & $3214(53.7)$ & $194(56.4)$ & $3020(53.5)$ & 0.300 \\
\hline female & $2772(46.3)$ & $150(43.6)$ & $2622(46.5)$ & \\
\hline Birth weight, $\mathrm{g}$ & $3202.8 \pm 458.7$ & $2368.5 \pm 520.9$ & $3257.7 \pm 402.2$ & $<0.001$ \\
\hline $\begin{array}{l}\text { Apolipoprotein } \\
\text { levels in } \\
\text { mid-pregnancy }\end{array}$ & & & & \\
\hline ApoA-1, g/L & $2.07(0.30)$ & $2.11(0.36)$ & $2.07(0.30)$ & 0.094 \\
\hline ApoB, g/L & $0.96(0.28)$ & $1.00(0.30)$ & $0.95(0.28)$ & $<0.001$ \\
\hline ApoB/ApoA-1 & $0.47(0.14)$ & $0.48(0.16)$ & $0.46(0.14)$ & 0.003 \\
\hline
\end{tabular}

Note: Data are presented as mean \pm SD for normally distributed data, median (IQR) for skewed data and numbers (percentage) for categorical data. BMI, pre-pregnancy body mass index; GDM, gestational diabetes mellitus; ApoA-1, apolipoprotein A-1; ApoB, apolipoprotein B.

Table 2. Odds ratios and $95 \%$ confidence intervals for preterm birth according to maternal ApoA-1, ApoB and ApoB/ApoA-1.

\begin{tabular}{llll}
\hline Variables & Total/preterm birth & Model 1 & Model 2 \\
ApoA-1 & & & \\
T1 & $1997 / 111$ & 1.00 (reference) & 1.00 (reference) \\
T2 & $2029 / 96$ & $0.84(0.64,1.12)$ & $0.86(0.65,1.15)$ \\
T3 & $1960 / 137$ & $1.28(0.99,1.66)$ & $1.25(0.96,1.64)$ \\
$P$ for trend & & 0.064 & 0.091 \\
Per 1-g/L increase in ApoA-1 & & $1.39(0.86,2.24)$ & $1.31(0.81,2.12)$ \\
ApoB & $2048 / 93$ & & \\
T1 & $1968 / 117$ & 1.00 (reference) & 1.00 (reference) \\
T2 & $1970 / 134$ & $1.33(1.01,1.76)$ & $1.26(0.95,1.68)$ \\
T3 & & $1.54(1.17,2.02)$ & $1.41(1.07,1.87)$ \\
$P$ for trend & & 0.002 & 0.017 \\
Per 1-g/L increase in ApoB & & $2.71(1.65,4.42)$ & $2.33(1.40,3.86)$ \\
ApoB/apoA-1 & $1995 / 101$ & $1.00($ reference) & $1.00($ reference) \\
T1 & $1996 / 103$ & $1.02(0.77,1.35)$ & $1.06(0.79,1.41)$ \\
T2 & $1995 / 140$ & $1.42(1.09,1.85)$ & $1.35(1.03,1.78)$ \\
T3 & & 0.007 & 0.024 \\
$P$ for trend & $5.55(2.04,14.99)$ & $4.10(1.47,11.36)$ \\
Per 1 increase in ApoB/ApoA-1 & & &
\end{tabular}

Note: $\mathrm{P}$ for trend were performed by assigning the median value of each category as a continuous variable in a separate model. Model 1: unadjusted. Model 2, adjusted for maternal pre-pregnancy BMI, age at delivery, parity, delivery mode, gestational diabetes mellitus and infant sex.

Table 3. Subgroup analyses of the associations between ApoB, ApoB/ApoA-1 and preterm birth, stratified by pre-pregnancy BMI, maternal age and parity.

\begin{tabular}{lllll}
\hline Subgroup & ApoB & ApoB & ApoB/ApoA-1 & ApoB/ApoA-1 \\
& OR (95\% CI) & $P$ for interaction & OR (95\% CI) & $P$ for interaction \\
overall & $2.33(1.40,3.86)$ & & $4.1(1.47,11.36)$ &
\end{tabular}


Pre-pregnant BMI, kg/ $\mathrm{m}^{2}$

$\begin{array}{lllll}<24 & 2.50(1.43,4.33) & 0.84 & 4.95(1.60,15.21) & 0.61 ? \dot{\iota} ? \\ 24 & 2.35(0.23,24.32) & & 2.35(0.23,24.32) & \\ \text { Maternal age at delivery, years } & & & & \\ <35 & 2.18(1.12,4.19) & 0.18 & 2.92(0.77,11.03) & 0.29 ? \dot{\iota} ? \\ 35 & 2.56(1.15,5.65) & & & \\ \text { Parity } & & & 1.20(0.19,7.41) & 0.27 \\ \text { Primiparous } & 1.66(0.66,4.10) & 0.33 & 7.27(2.12,24.82) & \\ \text { Multiparous } & 2.72(1.47,4.99) & & \end{array}$

Note: Adjusted for maternal pre-pregnancy BMI, age at delivery, parity, delivery mode, gestational diabetes mellitus and infant sex. Interactions across subgroups were tested by Wald test.

Table 4. Sensitivity analyses: odds ratios and $95 \%$ confidence intervals for preterm birth according to maternal serum ApoA-1, ApoB and ApoB/ApoA-1.

Sensitivity analyses

Excluding women with gestational diabetes mellitus $(\mathrm{n}=4,965)$

ApoA-1

ApoB

ApoB/ApoA-1

\section{Model 1}

Excluding women with gestational diabetes mellitus $(\mathrm{n}=4$

$1.20(0.70,2.06)$

$2.70(1.54,4.67)$

$6.21(2.01,18.97)$

Note: Model 1: unadjusted. Model 2: adjusted for maternal pre-pregnancy BMI, age at delivery, parity, delivery mode and infant sex. 


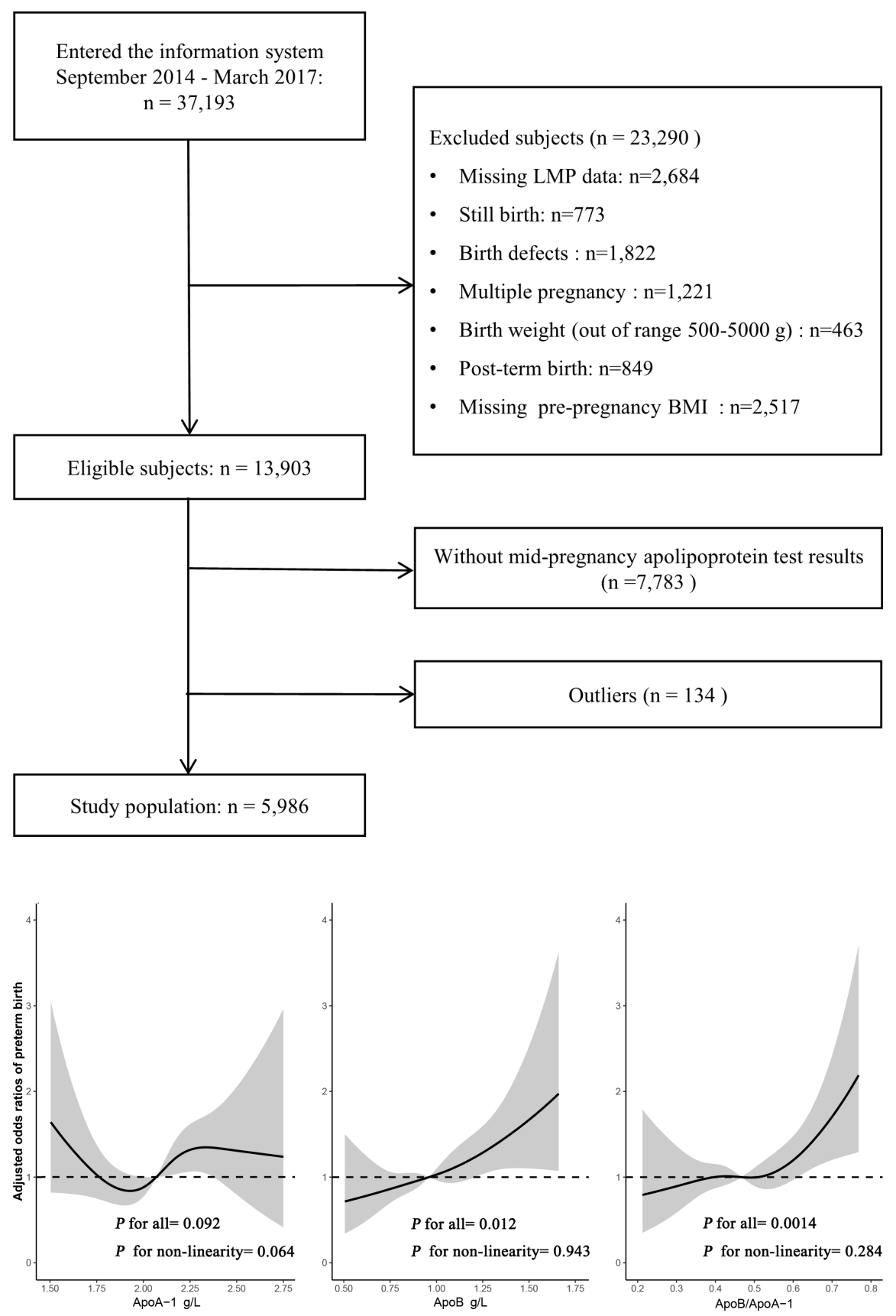

